\title{
ON MULTIPLICATIVE MAPPINGS OF RINGS OF OPERATORS
}

\author{
S. CATER
}

Let $L\left(h_{n}\right)$ be the aggregate of linear transformations (sometimes called operators) of the complex $n$ dimensional Hilbert space $h_{n}$ into itself. All members of $L\left(h_{n}\right)$ are bounded and we assign to $L\left(h_{n}\right)$ the topology induced by the usual metric. The determinant is a well defined mapping of $L\left(h_{n}\right)$ into the complex numbers (see [1]) which is continuous, multiplicative in the sense that $\operatorname{det}(A B)=(\operatorname{det} A)(\operatorname{det} B)$ for all $A, B \in L\left(h_{n}\right)$ and ${ }^{*}$ in the sense that $\operatorname{det}(c I) \geqq 0$ where $I$ is the identity mapping and $c$ is a nonnegative scalar.

It is our purpose to study the determinant and other continuous *-multiplicative mappings of $L\left(h_{n}\right)$ into the complex numbers. The functions identically 0 and identically 1 , for example, are such mappings other than the determinant. However we have the following uniqueness theorem. (In all that follows " $c$ " denotes the operator " $c I$ " where $c$ is scalar, $I$ is the identity; the distinction between the operator " $c$ " and the scalar " $c$ " can easily be inferred from the context.)

Theorem 1. Let $\phi$ be a continuous *-multiplicative mapping of $L\left(h_{n}\right)$ into the complex numbers such that $\phi(\exp (1+i))=\exp (n+n i)$. Then $\phi$ is the determinant on $L\left(h_{n}\right)$.

The problem of characterizing the determinant as a multiplicative mapping of the ring of $n$ by $n$ matrices with complex entries into the complex field is not new. In [3] Stephanos proved that such a multiplicative mapping which is differentiable with respect to each entry in the matrix (when all the other entries are fixed) must be a power of the determinant. Stephanos' theorem will be proved in our work also. (See [2] for a discussion of multiplicative mappings which are polynomials in the entries.) We will present seven lemmas before proving Theorem 1 . The first four show that $\phi$, like the determinant, is zero on singular operators.

Lemma 1. For the mapping given in Theorem $1, \phi(0)=0$.

Proof. We have $\phi(0)=\phi(\exp (1+i) 0)=\phi(\exp (1+i)) \phi(0)$ $=\exp (n+n i) \phi(0)$. But $\exp (n+n i) \neq 1$ and hence $\phi(0)=0$.

LEMMA 2. For the mapping $\phi$ given in Theorem $1, \phi(1)=1$.

Received by the editors October 20, 1960 and, in revised form, February 12, 1961. 
Proof. We have $\exp (n+n i)=\phi(\exp (1+i) 1)=\phi(\exp (1+i)) \phi(1)$ $=\exp (n+n i) \phi(1)$. But $\exp (n+n i) \neq 0$ and hence $\phi(1)=1$.

LEMMA 3. If $U \in L\left(h_{n}\right)$ is nonsingular, $\phi\left(U A U^{-1}\right)=\phi(A)$ for all $A \in L\left(h_{n}\right)$.

Proof. We have $\phi\left(U A U^{-1}\right)=\phi(U) \phi\left(A U^{-1}\right)=\phi\left(A U^{-1}\right) \phi(U)$ $=\phi\left(A U^{-1} U\right)=\phi(A)$.

Lemma 4. If $A \in L\left(h_{n}\right)$ is singular, then $\phi(A)=0$.

Proof. Select a unitary operator $U_{1}$ such that $U_{1} A U_{1}^{-1}$ annihilates a vector in the range of $A$. The dimension of the null space of $\left(U_{1} A U_{1}^{-1}\right) A$ is at least 2 . By induction there exist $n-1$ (or fewer) unitary operators $U_{1}, U_{2}, \cdots, U_{n-1}$ such that $\left(U_{n-1} A U_{n-1}^{-1}\right) \cdots$ $\left(U_{1} A U_{1}^{-1}\right) A=0$. By Lemmas 1,3 we have $0=\phi(0)=\phi(A)^{n}$ and $\phi(A)=0$.

Before we present the remaining lemmas we introduce the mapping $p$ as follows. Let $x$ be a nonzero vector and let $h_{n-1}$ be the orthogonal complement of $(x)$, the subspace spanned by $x$. For each scalar $a$ let $T_{a}$ denote the operator which is the identity on $h_{n-1}$ and carries $x$ into $a x$. Define $p(a)=\phi\left(T_{a}\right)$; by Lemma $3, p$ is independent of the choice of $x$.

In the next two lemmas we show that $\phi(A)=p(\operatorname{det} A)$ for $A \in L\left(h_{n}\right)$. This is already established on singular operators, both sides reducing to 0 . It is also evident on operators of the type $T_{a}$ described above.

Lemma 5. If $A \in L\left(h_{n}\right)$ is normal, $\phi(A)=p(\operatorname{det} A)$.

Proof. Let $A$ be normal. By the spectral theorem there exist operators $A_{1}, \cdots, A_{n}$ of the type $T_{a}$ described above such that $A=A_{1} \cdots A_{n}$. Then $\phi(A)=\phi\left(A_{1}\right) \cdots \phi\left(A_{n}\right)=p\left(\operatorname{det} A_{1}\right) \cdots$ $p\left(\operatorname{det} A_{n}\right)$. Observe that $p$ is multiplicative because $p(a b)=\phi\left(T_{a} T_{b}\right)$ $=\phi\left(T_{a}\right) \phi\left(T_{b}\right)=p(a) p(b)$. Hence $\phi(A)=p\left(\operatorname{det} A_{1} \cdots \operatorname{det} A_{n}\right)$ $=p(\operatorname{det} A)$.

Lemma 6. If $A \in L\left(h_{n}\right)$, then $\phi(A)=p(\operatorname{det} A)$.

Proof. It remains only to prove $\phi(A)=p(\operatorname{det} A)$ for $A$ nonsingular. By the polar decomposition there is a unitary operator $U$ such that $A=U\left(A^{*} A\right)^{1 / 2}$, where $A^{*}$ denotes the operator adjoint of $A$. But $U$ and $\left(A^{*} A\right)^{1 / 2}$ are normal, and hence $\phi(A)=\phi(U) \phi\left(\left(A^{*} A\right)^{1 / 2}\right)$ $=p(\operatorname{det} U) p\left(\operatorname{det}\left(A^{*} A\right)^{1 / 2}\right)=p\left(\operatorname{det} U \operatorname{det}\left(A^{*} A\right)^{1 / 2}\right)=p(\operatorname{det} A)$ by Lemma 5.

Having established Lemma 6 we investigate the mapping $p$. 
LEMMA 7. The mapping $p$ is continuous and multiplicative, carries zero into zero, carries the unit circle into itself and carries the positive real axis into itself.

Proof. Since $\phi$ is continuous, $p$ is continuous also. By Lemma 4, $p(0)=0$ and in Lemma 5 we showed that $p$ is multiplicative. For $|z|=1$ we have $p(z) \neq 0$; otherwise $1=\phi(1)=p\left(z z^{-1}\right)=p(z) p\left(z^{-1}\right)=0$. Hence $p$ is bounded away from zero on the unit circle. For $|z|=1$ we also have $|p(z)|=1$; otherwise $|p(z)| \neq 1,|z|=1$ and $p\left(z^{m}\right)=p(z)^{m}$ is not bounded away from zero as $m$ runs through all positive and negative integers. Hence $p$ carries the unit circle into itself. Since $\phi$ is ${ }^{*}, p(c) \geqq 0$ for $c \geqq 0$. But clearly $p(c) \neq 0$ for $c>0$ and consequently $p$ carries the positive real axis into itself.

A slight digression from our development proves Stephanos' theorem. Let $\phi$ be a multiplicative mapping of the ring of $n$ by $n$ matrices with complex entries into the complex plane such that $\phi$ is differentiable with respect to each entry. It follows that there is a multiplicative mapping $p$ of the complex plane into itself such that $\phi(A)$ $=p(\operatorname{det} A)$ for every $n$ by $n$ matrix $A$. By considering diagonal matrices for which all diagonal elements after the first are 1 , we see that $p$ is entire. Hence $p$ must be of the form $p(z)=z^{m}$ for some integer $m \geqq 0$ and $\phi(A)=(\operatorname{det} A)^{m}$. Indeed it suffices if $\phi$ is differentiable with respect to one diagonal entry.

To establish Theorem 1 we need only show that $p$ is the identity mapping.

Proof of Theorem 1 . Since $p$ is a continuous multiplicative mapping of the unit circle into itself it follows that there is an integer $m$, positive, negative or zero, such that $p(z)=z^{m}$ for $|z|=1$. Since $p$ is a continuous multiplicative mapping of the positive real axis into itself there is a real number $r$ such that $p(x)=x^{r}$ for $x>0$. Now by hypothesis $\phi(\exp (1+i))=\exp (n+n i)=p(\exp (n+n i))$ $=p(\exp n) p(\exp n i)$. But $|p(\exp n i)|=1$ and $p(\exp n)>0$; hence $\exp (n m i)=\exp (n i)$ and $\exp (r n)=\exp n$. Clearly $r=1$; because $\pi$ is irrational $m=1$. Hence $p$ is the identity and the proof is complete.

Next we turn to continuous ${ }^{*}$-multiplicative mappings other than the determinant.

TheOREM 2. Let $\phi$ be a continuous ${ }^{*}$-multiplicative mapping of $L\left(h_{n}\right)$ into the complex numbers. Then

(1) If $\phi(\exp (1+i))=0, \phi$ is identically 0 .

(2) If $|\phi(\exp (1+i))|=1, \phi$ is identically 1.

(3) If $\theta$ is another continuous *-multiplicative mapping such that 


$$
\phi(\exp (1+i))=\theta(\exp (1+i)),
$$

then $\phi=\theta$ on $L\left(h_{n}\right)$.

Proof of (1). If $\phi(\exp (1+i))=0$, then $\phi(A)=\phi(\exp (1+i))$ - $\phi(\exp (-1-i) A)=0$, all $A \in L\left(h_{n}\right)$.

ProOF of (2). Suppose $|\phi(\exp (1+i))|=1$ but there is a $T \in L\left(h_{n}\right)$ such that $\phi(T) \neq 1$. Then $\phi(0)=\phi(0) \phi(T)$ and $\phi(0)=0$. Lemma 1 is valid for $\phi$, and so are all the Lemmas 1-7. In the proof of Theorem 1 we necessarily have $r=0$, and hence $|\phi(A)|=1$ for $A$ nonsingular. But in every neighborhood of the zero operator there are nonsingular operators, contrary to the fact that $\phi$ is continuous. Hence $\phi$ is identically 1.

Proof of (3). We can suppose that $\phi(\exp (1+i))=\theta(\exp (1+i))$ is not 0 and not 1 . Then Lemmas 1-7 apply to $\phi$ and to $\theta$. In the proof of Theorem 1, $\phi$ and $\theta$ define the same $r$ and the same $m$, and the result is established.

Given a complex number $c$ there is at most one continuous *-multiplicative mapping $\phi$ of $L\left(h_{n}\right)$ into the complex plane such that $\phi(\exp (1+i))=c$. It remains to find the admissible values for $c$, those complex numbers for which there exists such a $\phi$. Clearly 0 and 1 are admissible values. Lemmas $1-7$ are valid for any $\phi$ not identically 0 or 1 and the proof of Theorem 1 shows that $\arg \phi(\exp (1+i))$ is a multiple of $n$. Likewise $|\phi(\exp (1+i))|>1$; otherwise $r \leqq 0$ and $\phi$ is discontinuous at the zero operator. This proves

Theorem 3. The admissible values of $c$ are 0, 1 and any complex number $c$ for which $|c|>1$ and $c /|c|$ is a (positive, negative or zero) power of $\exp (n i)$.

\section{REFERENCES}

1. P. R. Halmos, Finite dimensional vector spaces, $2 \mathrm{~d}$ ed., Van Nostrand, Princeton, N. J., 1958.

2. K. Hensel, Über den Zusammenhang zwischen den Systemen und iltren Determinanten, J. Reine Angew. Math. 159 (1928), 246.

3. C. Stephanos, Sur une propriêté caractéristique des déterminants, Ann. Mat. 21 (1913), 233-236.

UNIVERSITY OF OREGON 